November 7, 2017

Submitted to: Journal of Power Sources

\title{
Intelligent Energy Harvesting Scheme for Microbial Fuel Cells: \\ Maximum Power Point Tracking and Voltage Overshoot Avoidance
}

\author{
Muhannad Alaraj, Miloje Radenkovic, Jae-Do Park* \\ Department of Electrical Engineering \\ University of Colorado Denver, Denver, CO, 80217
}

*Corresponding author

Phone: (303) 352-3743; Fax: (303) 556-2383; Email: jaedo.park@ucdenver.edu 


\begin{abstract}
Microbial fuel cells (MFCs) are renewable and sustainable energy sources that can be used for various applications. The MFC output power depends on its biochemical conditions as well as the terminal operating points in terms of output voltage and current. There exists one operating point that gives the maximum possible power from the MFC, maximum power point (MPP), for a given operating condition. However, this MPP may vary and needs to be tracked in order to maintain the maximum power extraction from the MFC. Furthermore, MFC reactors often develop voltage overshoots that cause drastic drops in the terminal voltage, current, and the output power. When the voltage overshoot happens, an additional control measure is necessary as conventional MPPT algorithms will fail because of the change in the voltagecurrent relationship. In this paper, the extremum seeking (ES) algorithm was used to track the varying MPP and a voltage overshoot avoidance (VOA) algorithm is developed to manage the voltage overshoot conditions. The proposed ES-MPPT with VOA algorithm was able to extract 197.2mJ during 10-minute operation avoiding voltage overshoot, while the ES MPPT-only scheme stopped harvesting after only $18.75 \mathrm{~mJ}$ because of the voltage overshoot happened at 0.4 minute.
\end{abstract}

Keywords: Microbial fuel cell, maximum power point tracking, extremum seeking control, voltage overshoot 


\section{Introduction}

Microbial fuel cells (MFCs) generate electricity using the bacteria that exists in waste water and marine sediment. Electrochemically active bacteria (EAB), such as Shewanella and Geobacter, can transport electrons extracellularly to the anode of the MFC, while consuming organic matter in the anode chamber. Transporting electrons from the anode to the cathode through external circuit results in a direct current flowing to the external electrical load [1-3]. The electrical power available from MFC is low compared to other types of fuel cell such as hydrogen fuel cells, mainly because of the difficulty in scaling up. The maximum power density reported up to date is $19 \mathrm{~W} / \mathrm{m}^{2}$ [4]. However, they have been investigated as a potential renewable energy source to power some applications such as remote wireless sensors in underwater systems where it is costly to replace batteries [5-7].

The current and voltage at MFC terminals are inversely proportional typically in the ohmic region where reactors are operated for power generation. Drawing more current reduces the terminal voltage of MFC and vice versa because of the voltage drop across the internal resistance of MFC [8,9]. This behavior results in a quadratic power characteristic, where the power from the MFC can be maximized when a specific amount of current is drawn. It can be shown that this maximum power operation can be achieved when the external resistance equals to the internal resistance [2].

Operating at the maximum power point (MPP) means that the highest amount of attainable instantaneous power can be extracted in any given condition. For the MFC, there are other advantages of MPP operation such as reduction of MFC's start-up time compared to fixed resistance operation [10,11], improvement of columbic efficiency and waste water treatment efficiency $[12,13]$, selection of electro-active bacteria within complex microbiomes 
[14], and avoidance of voltage reversal phenomena in stacked MFC systems [15].

Various maximum power point tracking (MPPT) algorithms have been investigated to track the varying MPP [13,16-18]. Perturb and observe (P\&O) is one of the basic MPPT algorithms, which applies a small voltage perturbation to the MFC output terminals and observes the output power. By comparing the previous and current output, it is straightforward to decide the direction of next step that would increase the power towards MPP. Tracking MPP has been achieved using load resistors $[13,16]$ or power electronics converters $[17,18]$. Although the resistor-based methods can track the MPP, it should be noted that using resistance at the MFC output is meaningless from practical perspectives because the extracted energy will be dissipated as heat rather than stored and used to support a load. Power electronics converters and charge pumps have been investigated to extract and store the energy in a capacitor or a battery [17-21].

In this study, an extremum seeking (ES) algorithm is used in conjunction with a power electronics converter to extract the maximum power from MFC and track the varying MPP. The ES algorithm finds the MPP by applying sinusoidal voltage perturbation and observing the change in power from which the direction to MPP is determined. Although the ES algorithm and $\mathrm{P} \& \mathrm{O}$ technique share the idea of voltage perturbation and power change observation, they differ in the type of perturbation. The ES algorithm applies a continuous sinusoidal voltage perturbation that generates a sinusoidal component as well as a DC component in the MFC output current and power, which will result in either positive or negative change in powercurrent product that can be used to track the varying MPP.

One of the problems that could happen when extracting energy from MFC is voltage overshoot [22-24]. When high current is extracted from MFC, the anode potential changes 
from being negative to be positive, which significantly reduces the terminal voltage of MFC as well as the output power [23]. The voltage overshoot could prohibit operating at the theoretical MPP if it happens at or before the MPP. The existing MPPT algorithms are not able to operate at the correct operating point under the voltage overshoot condition because, once the voltage overshoot point is reached, the power characteristic is no longer quadratic as the slope of MFC's voltage-current characteristic becomes positive. If the algorithm tries to increase the current to increase power, the voltage decreases so that the power keeps decreasing to a low level or even to zero. Research has been focused on understanding and eliminating the voltage overshoot problem [22-26], rather than maintaining operation in the presence of voltage overshoot. In this study, an energy harvesting algorithm that can manage the voltage overshoot condition is also developed.

The proposed intelligent MFC energy management scheme can detect and avoid the voltage overshoot, and maintain the operating point at a sustainable level to continue harvesting energy from MFC, as well as track the varying MPP in normal conditions.

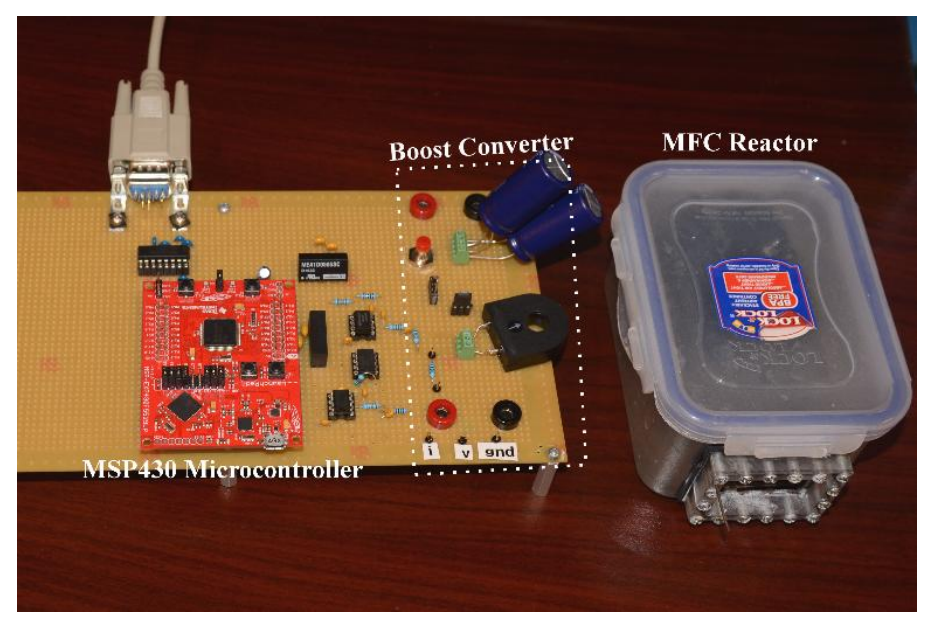

Fig. 1. Experimental setup: controller, power converter, and MFC reactor. 


\section{Methods and Materials}

2.1. Microbial fuel cell

A single-chamber air-cathode MFC reactor was built in the lab as can be seen in Fig. 1 using a 450mL food container (HPL807, Lock \& Lock), a 2" (5.08cm)-diameter brush anode (\#313239, Mill-Rose Company, OH), and 1.5"×1" $(3.81 \mathrm{~cm} \times 2.54 \mathrm{~cm})$ 5-layer hydrogen-air membrane electrode assembly (HA5-MEA, Fuel Cells Etc, TX). The MEA has a catalyst layer (60 wt\% Pt on Vulcan, $0.5 \mathrm{mg} / \mathrm{cm}^{2}$ ) on each side of the 2-mil proton exchange membrane (Nafion 212). It also has a 410 micron gas diffusion layer in both sides. The cathode electrode has a small piece of 1-mm titanium wire (45485-BY, Alfa Aesar, MA). To hold the cathode MEA and the titanium wire, two rubber gaskets and two $2 " \times 1.5 "(5.08 \mathrm{~cm} \times 3.81 \mathrm{~cm})$ polycarbonate brackets fabricated by a machine shop used. The two rubber gaskets hold the cathode MEA from both sides to avoid any leak and the whole cathode electrode assembly were held together by $1.5 "(1.27 \mathrm{~cm}) 4-40$ aluminum screws.

The reactor was inoculated with sludge from a local water treatment facility and a medium that consists of $2 \mathrm{~g} / \mathrm{L} \mathrm{CH}_{3} \mathrm{COONa}, 0.31 \mathrm{~g} / \mathrm{L} \mathrm{NH} \mathrm{NH}_{4}, 0.13 \mathrm{~g} / \mathrm{L} \mathrm{KCl}, 3.321 \mathrm{~g} / \mathrm{L}$ $\mathrm{NaH}_{2} \mathrm{PO}_{4} \cdot 2 \mathrm{H}_{2} \mathrm{O}$, and $10.317 \mathrm{~g} / \mathrm{L} \mathrm{Na} 2 \mathrm{HPO}_{4} \cdot 12 \mathrm{H}_{2} \mathrm{O}$ was used. The reactor was operated in fedbatch mode and the solution was replaced when necessary (around six days on average) until the terminal voltage stabilized at around $450 \mathrm{mV}$ with a $1 \mathrm{k} \Omega$ resistor load.

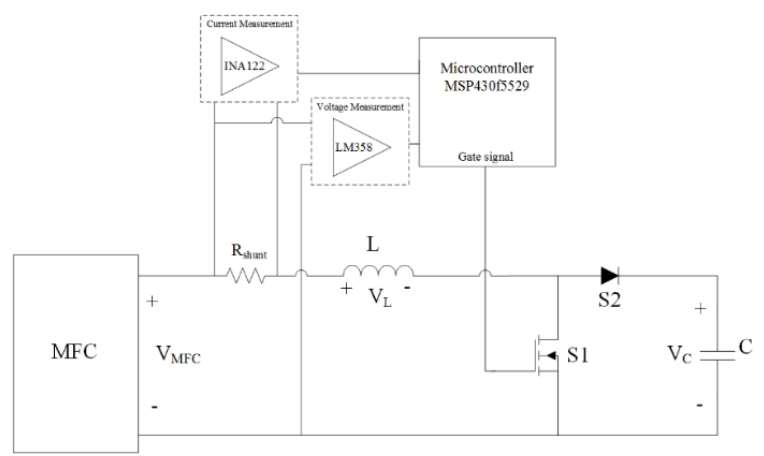

Fig. 2. Schematic diagram of harvesting controller. 
2.2. Power conversion hardware

To test the proposed algorithms, the boost converter topology, shown in Fig. 2, was chosen because of its simplicity. The operation of the boost converter consists of two modes. The first mode is when the switch $S_{1}$ is closed while the switch $S_{2}$ is open, in which the current flows through the inductor only. In this mode, the inductor voltage becomes $V_{M F C}$, and the inductor current, i.e., the MFC current, should follow the inductor constitutive equation

$v_{l}(t)=L \frac{d i_{i}(t)}{d t}$

where $v_{l}$ is the voltage across the inductor, $i_{l}$ is the current through the inductor, and $L$ is the inductance of the inductor. In this mode, the inductor stores the energy from the MFC, which is given as

$\mathrm{E}=\frac{1}{2} L i_{l}^{2}$

The second mode is complimentary to the first mode: $S_{1}$ is open and $S_{2}$ is closed. The current will flow through $S_{2}$ to the output capacitor. This will cause the inductor voltage polarity to be reversed and its value will depend on the rate of change of the inductor current as shown in (1). Typically, the inductor voltage sharply increases at the beginning, then exponentially decreases as the stored energy (2) is discharged to the capacitor. The inductor voltage will be added on top of MFC output voltage, which generates high voltage to charge the output capacitor. The boost converter was built using a MOSFET (4906 NG, ON Semiconductor, AZ) as $S_{1}$, a diode (BAT46, Vishay Electronic $\mathrm{GmbH}$ ) as $S_{2}$, and an inductor (CST206-1A, Triad Magnetics, CA) that has $28 \mathrm{mH}$ at $1 \mathrm{kHz}$.

A microcontroller (MSP430F5529, Texas Instruments, TX) is used to implement the algorithms and to drive the boost converter. Its built-in analog-to-digital converter (ADC) reads 
MFC voltage and current. An instrumentation amplifier (INA122, Texas Instruments, TX) measures the MFC current using a shunt resistor that is connected in series with the inductor. A $10 \Omega$ shunt resistor was selected to minimize the effect on voltage measurement, while having enough resolution for current measurement. The INA122 amplifies the voltage drop across shunt resistor and sends it to microcontroller's ADC. The implementation of controller and boost converter, and the functional block diagram can be seen in Figs. 1 and 2, respectively.

\subsection{Extremum seeking control}

Often in engineering systems there is a need for optimizing the steady-state behavior by operating the system at its extreme (minimum or maximum) value. This objective can be accomplished by adjusting certain control input so that a desired performance criterion achieves and maintains its extreme value. Algorithms capable of performing such tasks is called an "extremum control algorithms", or an "extremum seeking (ES) algorithm". A chronological overview of the ES control developments along with a large number of references can be found in [27].

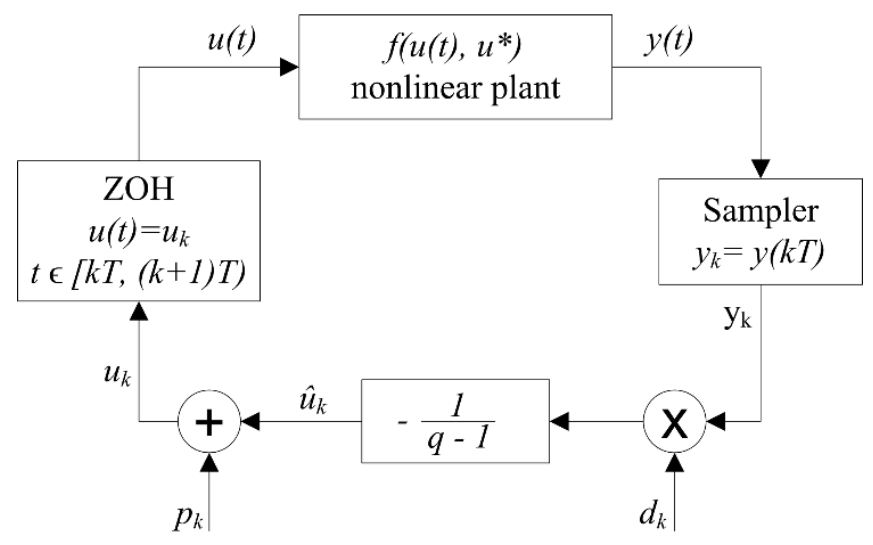

Fig. 3. Extremum seeking algorithm block diagram. 
Assume that the unknown steady-state input-output nonlinear map is described by

$y(t)=f\left(u(t), u^{*}\right), t \geq 0$

where $y(t)$ is measured output, $u(t)$ is the control input, and $u^{*}$ is the unknown parameter. We also assume that $f(\because$,$) achieves its extremum y^{*}$ when $u(t)=u^{*}$, i.e., $y^{*}=f\left(u^{*}, u^{*}\right)$. The functional block diagram of the ES control is shown in Fig. 3, where $y_{k}$ and $u_{k}, k=0,1, \cdots$, are discrete time sampled values of the output $y(t)$ and the input $u(t)$, respectively, while $q$ is the forward shift operator, i.e., $q u_{k}=u_{k+1}$. Here, $\mathrm{ZOH}$ represents the zero order hold operation defined by $u(t)=u_{k}$ for $t \in[k T,(k+1) T), k>0$, where $T$ is the sampling period. In Fig. $3, \hat{u}_{k}$ is an estimate of the unknown optimizer $u^{*}, p_{k}$ is the perturbation sequence added to $\hat{u}_{k}$, and $d_{k}$ is the demodulation signal. In this process, the amplitude of $p_{k}$ will be modulated by the slope of the nonlinear function $f(\because$,$) . The role of the signal d_{k}, k \geq 0$ is to produce the estimate of the gradient of $f(\because$,$) by demodulating the output sequence \left\{y_{k}\right\}$.

Similar to most ES literature, sinusoidal perturbation and demodulation signals are used in this paper.

$p_{k}=A \cos \left(\omega_{0} k\right), d_{k}=\mu A \cos \left(\omega_{0} k+\phi\right)$

where $k=0,1, \cdots, A>0, \mu>0$ and $\phi$ denotes an arbitrary phase angle shift. If $f(\cdot, \cdot)$ is a part of some system dynamics, the perturbation frequency $\omega_{0}$ should fall within the bandwidth of the system and a better estimate of the gradient of $f(\cdot$,$) can be obtained if \omega_{0}$ is higher $[28,29]$. Assuming that $f(\cdot$,$) has a maximum at u=u^{*}$, from Fig. 3 we can write

$\hat{u}_{k+1}=\hat{u}_{k}-d_{k} y_{k}, \quad k \geq 0$.

In case $f(\because$,$) is a quadratic function, f\left(u(t), u^{*}\right)=f_{0}+f_{1}\left(u(t)-u^{*}\right)^{2}, f_{1}>0$, the initial $\hat{u}_{0}$ is taken to be arbitrary. Although the iterate $u_{k}$ is closer to the optimal point $u^{*}$ if the perturbation amplitude $A$ is smaller, the analysis in [31] indicates that smaller $A$ results in a 


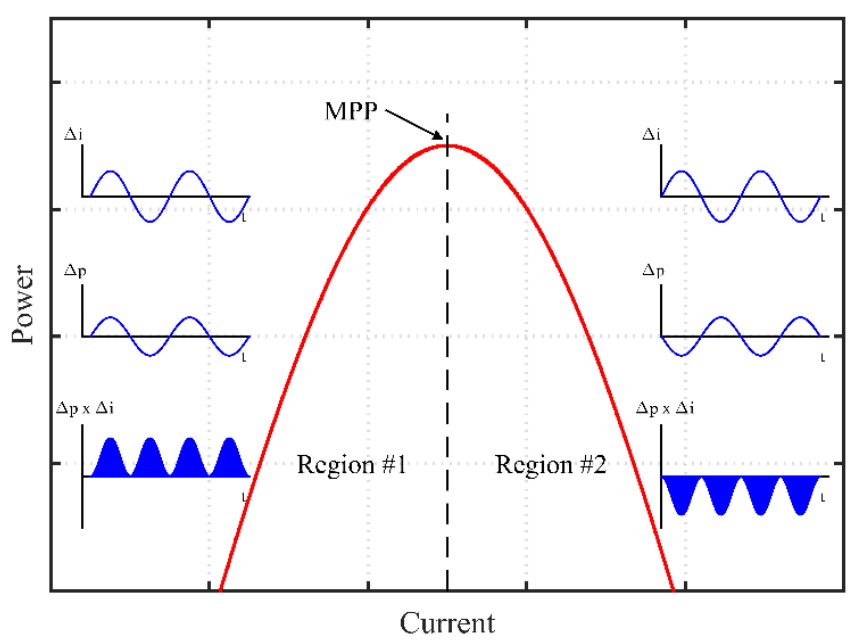

Fig. 4. Visual explanation of MPPT using ES algorithm

slower convergence of $\left\{\hat{u}_{k}\right\}$. The choice of $A$ is a trade-off between the rate of convergence of $u_{k}$ and the size of the error $u_{k}-u^{*}$. It can be shown that with (4), the algorithm in Fig. 3 gives $\left(u_{k}-u^{*}\right)^{2}=O\left(A^{2}\right)$.

In this paper, the instantaneous output power $p(t)$ and boost converter's duty ratio $D(t)$ are used as the system output $y(t)$ and input $u(t)$, respectively. Applying sinusoidal perturbation $p_{k}$ to the boost converter duty ratio $D(t)$ will create a sinusoidal perturbation in the MFC terminal voltage and, in turn, current. The demodulation signal $d_{k}$ is a function that is implemented numerically in the microcontroller to be used in the algorithm. The parameters for the perturbation and demodulation signals in (4) are $A=0.05, \omega_{0}=88.8 \mathrm{rad} / \mathrm{s}, \mu=18, \phi=$ $106^{\circ}$, and $T=0.33 \mathrm{~ms}$.

\subsection{Maximum power point tracking}

Fig. 4 shows a typical power-current curve of an MFC that has a linear voltage-current relationship. In region \#1 of the curve, the changes in current and power are in the same 
direction: increasing or decreasing the current increases or decreases the power, respectively. Therefore, when the MFC is sinusoidally perturbed by ES algorithm, the power will have a sinusoidal component in phase with that of the current. Integrating the product of the sinusoidal components of current and power will result in a positive value, which indicates the positive slope of power curve toward the MPP. The instantaneous product of the sinusoidal current and power components can be given as follows.

$\int i_{\Delta}(t) \times p_{\Delta}(t) d t=\int I_{\Delta} \cos \omega_{0} t \times P_{\Delta} \cos \omega_{0} t d t=\int \frac{I_{\Delta} P_{\Delta}}{2}\left(1+\cos 2 \omega_{0} t\right) d t>0$

where, $I_{\Delta}$ and $P_{\Delta}$ denotes the magnitude of instantaneous sinusoidal components of current $i_{\Delta}(t)$ and power $p_{\Delta}(t)$, respectively. The same scheme applies to region \#2 of the power curve, but in this region the current and the power will be out of phase, i.e., $180^{\circ}$ phase shift between two sinusoidal components, and integration of the current-power product will result in a negative value meaning negative slope after the MPP. Hence, the algorithm will know the power slope regardless where the algorithm starts; then it is straightforward to move towards the MPP and maintain operation at the MPP. This scheme was implemented with the ES algorithm described in Section 2.3.

2.5. Voltage overshoot avoidance (VOA) algorithm

One of the main changes in the electrical side of MFCs caused by the voltage overshoot happens in the relationship between output voltage and current. In normal operating conditions, MFC's terminal voltage and current change in opposite directions, where decreasing output voltage will increase the output current and vice versa. However, in the voltage overshoot region, they both increase or decrease at the same time. The polarization curves in Fig. 5 show the terminal voltage-current relationships in normal and voltage 


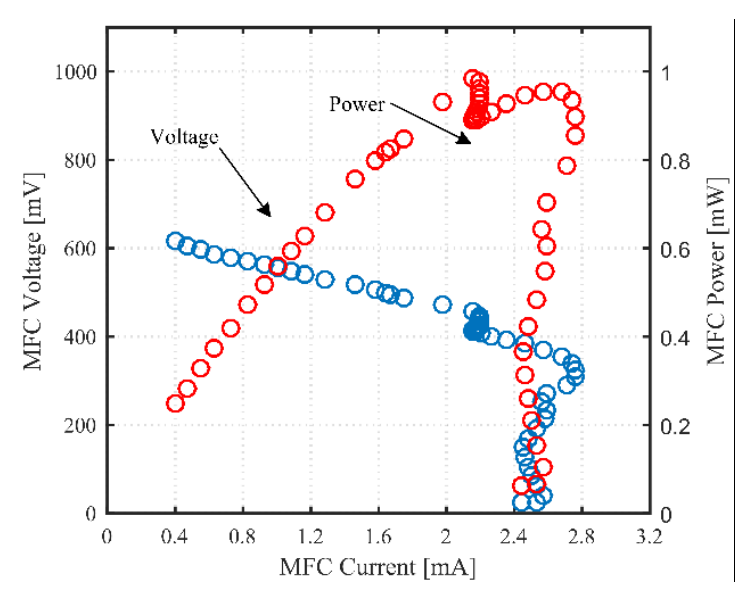

(a)

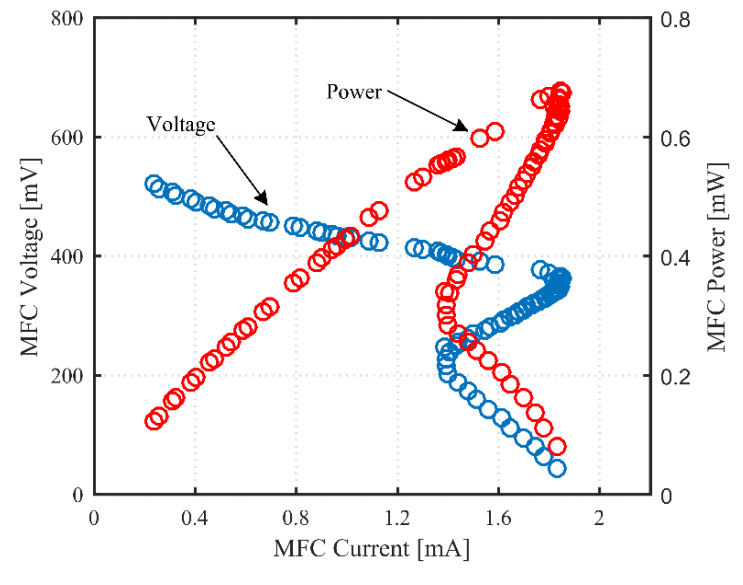

(b)

Fig. 5. MFC polarization curve with voltage overshoot. (a) Not recovered from voltage overshoot. (b) Slope of polarization curve is recovered to negative after voltage overshoot.

overshoot conditions.

This change in the voltage-current relationship makes the MPPT algorithms ineffective in the voltage overshoot region. The ES MPPT algorithm recognizes the voltage overshoot condition as the system is in region \#1 of Fig. 4 because the current and power change in the same direction. But, even if the algorithm increases the duty cycle of the boost converter trying to increase the current, the output current decreases as well as the output voltage. Consequently, the output power drops and the algorithm keeps increasing the duty cycle, which becomes a runaway condition. This continues until the power decreases to zero (Fig. 5 (a) case) as the duty cycle becomes one, or the system runs at a certain low power operating point if the slope of voltage-current characteristic gets back to negative before the duty cycle reaches one (Fig. 5 (b) case).

To detect the voltage overshoot while running the ES MPPT scheme, the change in voltage-current relationship, i.e., from changing in opposite directions to same direction, is 
used. Once the VOA algorithm detects the voltage overshoot, it interrupts the ES MPPT algorithm, records the last duty cycle, and opens the circuit. After waiting for the reactor to be stabilized at a high voltage, e.g., OCV, the ES MPPT algorithm is restarted with a duty cycle limit that is lower than the recorded one. If the overshoot happens again before the duty cycle hits the limit, the VOA algorithm repeats the cycle with further reduced duty cycle limit. The algorithm keeps reducing the limit until no overshoot occurs, so that continuous energy harvesting can be maintained at a sustainable voltage without system collapse. Fig. 6 shows the flow chart for the VOA algorithm.

\section{Experimental Results}

\subsection{MPPT}

Theoretical MPP occurs at the half of the OCV [2]. It can be seen from Fig. 5 that the voltage overshoot happens slightly before the theoretical MPP in the MFC reactor used in this paper. Hence, the output voltage collapses even before the MPPT algorithm finds the MPP, which makes evaluating the ES MPPT impossible. In order to evaluate the proposed MPPT algorithm, an equivalent circuit of the MFC [20] was used to simulate the behavior of the MFC. The equivalent circuit consists of a DC voltage source (GPC-30300, GW INSTEK) and a generic resistance in series. The DC voltage source was set to $700 \mathrm{mV}$ which is close the open circuit of MFC and a $151 \Omega$ resistor was used to extract the power that is comparable to the typical MFC's output power. The theoretical polarization curve of the equivalent circuit can be calculated using

$P=\left(V_{\text {int }}-i R_{\text {int }}\right) i$

where $V_{\text {int }}$ is the voltage of the DC power supply, $R_{\text {int }}$ is the resistance of the resistor, and $i$ is 
the output current. The dotted line in Fig. 7 (a) represents the theoretical polarization curve using (7). It can be seen that the ES MPPT algorithm successfully reaches to the MPP and maintains the operation there at the level of $0.81 \mathrm{~mW}$, which is the theoretical maximum power. The algorithm started from the low current and low power side of the polarization curve and kept increasing the current until the MPP was reached. 


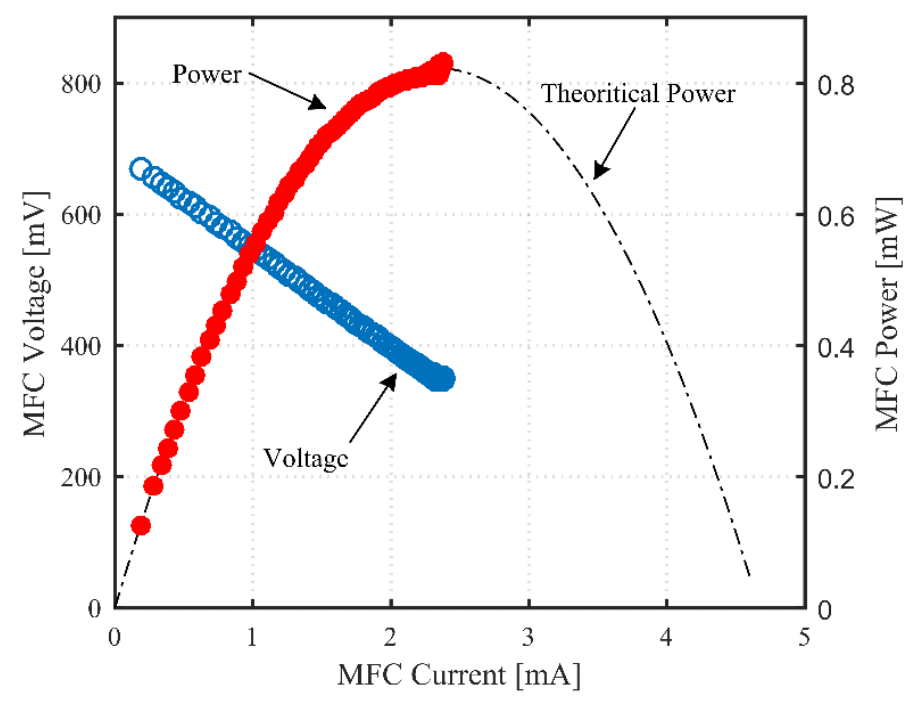

(a)

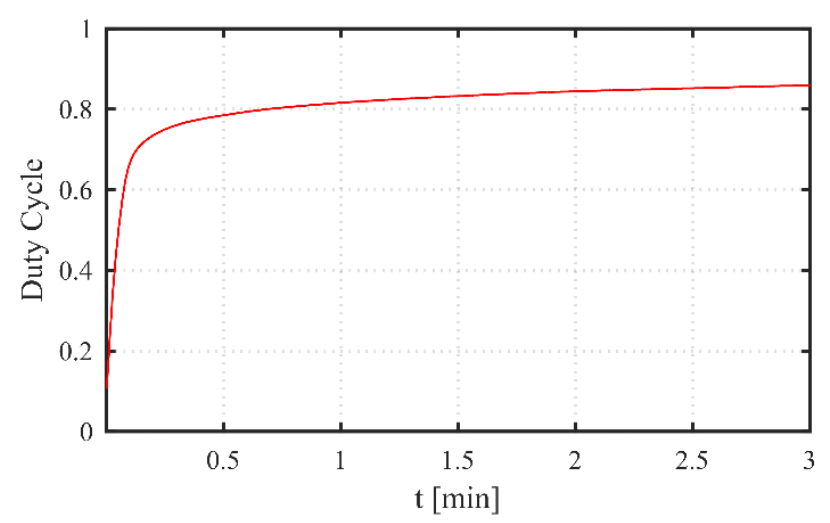

(b)

Fig. 7. ES MPPT experiment results. (a) Measured power on top of the theoretical curve and measured voltage. (b) Boost converter duty cycle.

The empty and filled circles in Fig. 7 (a) represent the voltage/current measurements and calculated output power, respectively. The duty cycle of the boost converter is shown in Fig. 7 (b). It can be noted that the duty cycle of the boost converter kept increasing after finding the MPP; it is because of the increasing output capacitor voltage. The ES MPPT algorithm keeps increasing the duty cycle to maintain the value of the current that gives the 
maximum power.

\subsection{Voltage overshoot avoidance}

The MFC reactor with the polarization characteristics in Fig. 5 was used to test the proposed VOA algorithm. For the sake of comparison, two experiments were conducted and each lasts for 10 minutes. The proposed VOA algorithm was enabled along with ES MPPT in the first experiment and disabled in the second experiment. The results show a substantial difference: the VOA algorithm operates the system at an operating point right before the voltage overshoot point, while the output power of the MPPT only case eventually decreased to zero.

The duty cycle of the boost converter for the two experiments is shown in Fig. 8. When the algorithm detected the overshoot around $0.4 \mathrm{~min}$, it interrupted the current extraction to prevent the MFC voltage from collapsing. Then, the algorithm waited for the MFC voltage to recover to a set point, $500 \mathrm{mV}$ in this work, and started running the $\mathrm{ES}$ algorithm again with a duty cycle limit. Since the new duty cycle limit is close to the duty cycle at the voltage overshoot trigger point, the voltage overshoot occurred again shortly after the power extraction restarted. The algorithm kept decreasing the duty cycle limit until it found one that stops the voltage overshoot. On the other hand, the ES MPPT kept running continuously after the voltage overshoot, as can be seen in Fig. 8, until the duty cycle reached one, the terminals were short-circuited, and the output power dropped to zero. 

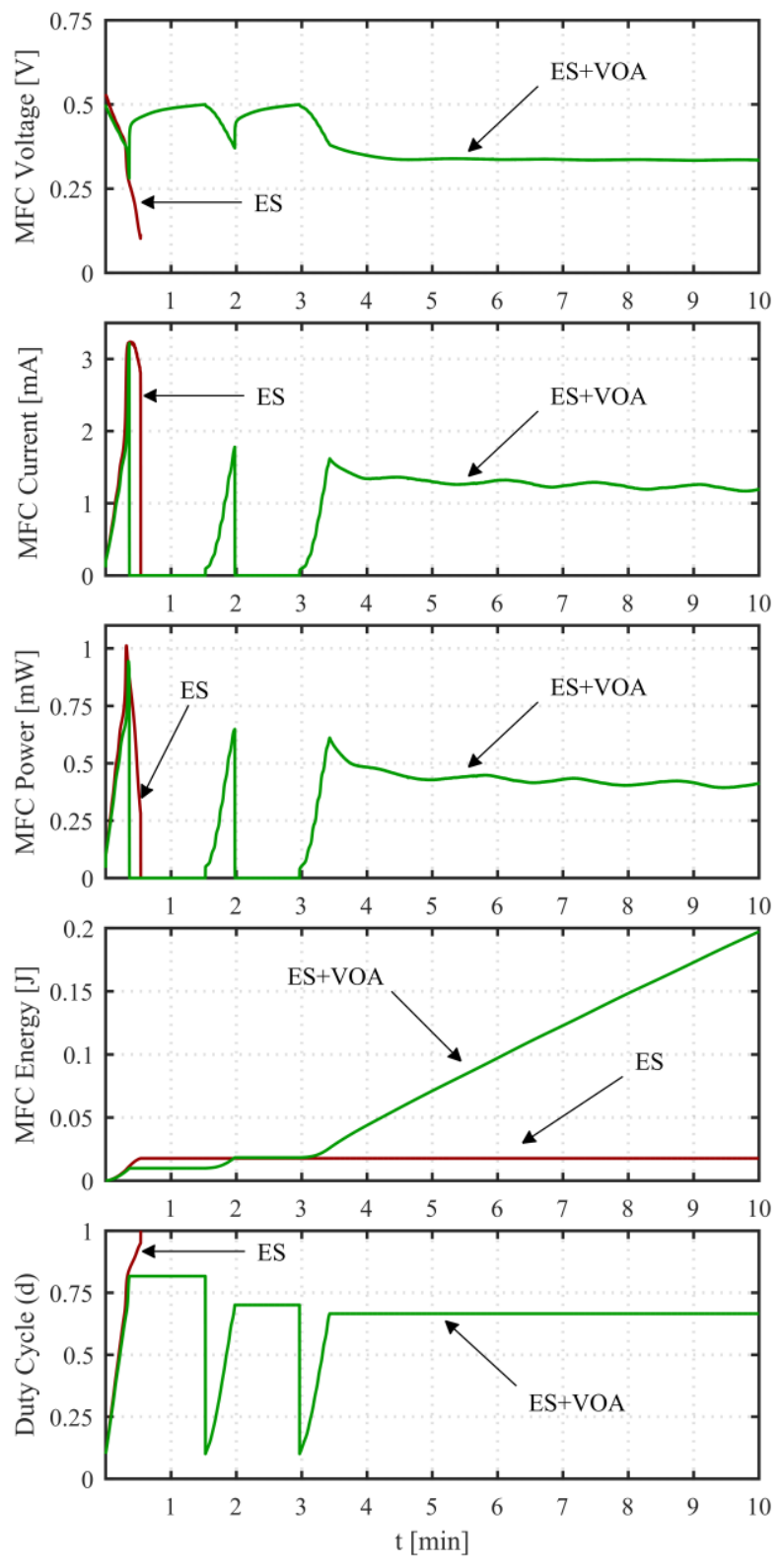

Fig. 8. Experimental results for MPPT-only and VOA algorithms under voltage overshoot condition: from top, MFC voltage, MFC current, MFC power, MFC energy, and duty cycle.

The energy that was harvested using the VOA algorithm was significantly higher compared to that in the ES MPPT only case. The power of the MFC went to zero around 0.4 min, because the VOA algorithm interrupted the current when the voltage overshoot was 
detected. On the other hand, the power decreased after the voltage overshoot around 0.4 min in ES MPPT only case. Eventually, the VOA algorithm was able to stably run the system at a high power generating operating point compared to zero power from the MPPT only case. With the MFC reactor used in this work, the VOA algorithm was able to extract $197.2 \mathrm{~mJ}$ during the 10-minute operation. While trying to find MPP without VOA algorithm, the reactor generated only $18.75 \mathrm{~mJ}$ before it collapsed. In terms of power, the ES MPPT-only algorithm extracted up to about $1 \mathrm{~mW}$ but it couldn't recover from the voltage overshoot, while ES and VOA operates at $0.45 \mathrm{~mW}$ in average but much longer.

\section{Conclusion}

The proposed ES MPPT algorithm showed simple and fast way to track the MPP using sinusoidal perturbations. However, if voltage overshoot happens, it is unable to maintain the operation because of the change in MFC's voltage-current characteristic. Once the power starts to drop due to the voltage overshoot, it is impossible to operate at the normal MPP or even

close to it. In this paper, a VOA algorithm was developed in conjunction with the ES MPPT to sustain the continuous energy extraction at an optimal operation point for a given condition. The experimental result demonstrated successful performance of the proposed intelligent MFC energy harvesting scheme that extracts much higher power and energy continuously even in presence of the voltage overshoot phenomenon.

\section{Acknowledgements}

This work was supported by the Office of Naval Research (ONR) and the National Science Foundation (NSF) under Award N000141512570 and 1511568, respectively. 


\section{References}

[1] H. Liu, B.E. Logan, Electricity generation using an air-cathode single chamber microbial fuel cell in the presence and absence of a proton exchange membrane., Environ. Sci. Technol. 38 (2004) 4040-4046. doi:Doi 10.1021/Es0499344.

[2] B.E. Logan, B. Hamelers, R. Rozendal, U. Schroder, J. Keller, S. Freguia, P. Aelterman, W. Verstraete, K. Rabaey, Microbial fuel cells: Methodology and technology, Environ. Sci. Technol. 40 (2006) 5181-5192. doi:10.1021/es0605016.

[3] Z. Du, H. Li, T. Gu, A state of the art review on microbial fuel cells: A promising technology for wastewater treatment and bioenergy, Biotechnol. Adv. 25 (2007) 464482. doi:10.1016/j.biotechadv.2007.05.004.

[4] H. Wang, J. Park, Z.J. Ren, Practical Energy Harvesting for Microbial Fuel Cells: A Review., Environ. Sci. Technol. 49 (2015) 3267-3277. doi:10.1021/es5047765.

[5] C. Donovan, A. Dewan, H. Peng, D. Heo, H. Beyenal, Power management system for a 2.5 W remote sensor powered by a sediment microbial fuel cell, J. Power Sources. 196 (2011) 1171-1177. doi:10.1016/j.jpowsour.2010.08.099.

[6] F. Zhang, L. Tian, Z. He, Powering a wireless temperature sensor using sediment microbial fuel cells with vertical arrangement of electrodes, J. Power Sources. 196 (2011) 9568-9573. doi:10.1016/j.jpowsour.2011.07.037. 
[7] Q. Zheng, L. Xiong, B. Mo, W. Lu, S. Kim, Z. Wang, Temperature and Humidity Sensor Powered by an Individual Microbial Fuel Cell in a Power Management System, Sensors. 15 (2015) 23126-23144. doi:10.3390/s150923126.

[8] P. Liang, X. Huang, M.Z. Fan, X.X. Cao, C. Wang, Composition and distribution of internal resistance in three types of microbial fuel cells, Appl. Microbiol. Biotechnol. 77 (2007) 551-558. doi:10.1007/s00253-007-1193-4.

[9] Y. Fan, E. Sharbrough, H. Liu, Quantification of the Internal Resistance Distribution of Microbial Fuel Cells Quantification of the Internal Resistance Distribution of Microbial Fuel Cells, Environ. Sci. Technol. 42 (2008) 8101-8107. doi:10.1021/es801229j.

[10] H.C. Boghani, J.R. Kim, R.M. Dinsdale, A.J. Guwy, G.C. Premier, Control of power sourced from a microbial fuel cell reduces its start-up time and increases bioelectrochemical activity, Bioresour. Technol. 140 (2013) 277-285. doi:10.1016/j.biortech.2013.04.087.

[11] D. Molognoni, S. Puig, M.D. Balaguer, A. Liberale, A.G. Capodaglio, A. Callegari, J. Colprim, Reducing start-up time and minimizing energy losses of Microbial Fuel Cells using Maximum Power Point Tracking strategy, J. Power Sources. 269 (2014) 403-411. doi:10.1016/j.jpowsour.2014.07.033.

[12] D. Molognoni, S. Puig, M.D. Balaguer, A.G. Capodaglio, A. Callegari, J. Colprim, Multiparametric control for enhanced biofilm selection in microbial fuel cells, J. Chem. Technol. Biotechnol. 91 (2016) 1720-1727. doi:10.1002/jctb.4760.

[13] R.P. Pinto, B. Srinivasan, S.R. Uiot, B. Tartakovsky, The effect of real-time external 
resistance optimization on microbial fuel cell performance, Water Res. 45 (2011) 15711578. doi:10.1016/j.watres.2010.11.033.

[14] A. Vilajeliu-Pons, L. Bañeras, S. Puig, D. Molognoni, A. Vilà-Rovira, E. Hernández-del Amo, M.D. Balaguer, J. Colprim, External Resistances Applied to MFC Affect Core Microbiome and Swine Manure Treatment Efficiencies, PLoS One. 11 (2016) e0164044. doi:10.1371/journal.pone.0164044.

[15] H.C. Boghani, G. Papaharalabos, I. Michie, K.R. Fradler, R.M. Dinsdale, A.J. Guwy, I. Ieropoulos, J. Greenman, G.C. Premier, Controlling for peak power extraction from microbial fuel cells can increase stack voltage and avoid cell reversal, J. Power Sources. 269 (2014) 363-369. doi:10.1016/j.jpowsour.2014.06.059.

[16] N. Degrenne, F. Buret, B. Allard, P. Bevilacqua, Electrical energy generation from a large number of microbial fuel cells operating at maximum power point electrical load, J. Power Sources. 205 (2012) 188-193. doi:10.1016/j.jpowsour.2012.01.082.

[17] J.-D. Park, Z. Ren, Hysteresis controller based maximum power point tracking energy harvesting system for microbial fuel cells, J. Power Sources. 205 (2012) 151-156. doi:10.1016/j.jpowsour.2012.01.053.

[18] H. Wang, J. Do Park, Z. Ren, Active energy harvesting from microbial fuel cells at the maximum power point without using resistors, Environ. Sci. Technol. 46 (2012) 52475252. doi:10.1021/es300313d.

[19] N. Degrenne, F. Morel, S. Adami, B. Allard, A. Zaoui, Self-Starting DC:DC Boost Converter for Low-Power and Low-Voltage Microbial Electric Generators, (2011) 889_- 
896.

[20] J. Park, Z. Ren, Efficient Energy Harvester for Microbial Fuel Cells using DC / DC Converters, IEEE Energy Convers. Congr. Expo. (2011) 3852-3858.

[21] H. Wang, Z. Ren, J. Do Park, Power electronic converters for microbial fuel cell energy extraction: Effects of inductance, duty ratio, and switching frequency, J. Power Sources. 220 (2012) 89-94. doi:10.1016/j.jpowsour.2012.07.092.

[22] P.C. Nien, C.Y. Lee, K.C. Ho, S.S. Adav, L. Liu, A. Wang, N. Ren, D.J. Lee, Power overshoot in two-chambered microbial fuel cell (MFC), Bioresour. Technol. 102 (2011) 4742-4746. doi:10.1016/j.biortech.2010.12.015.

[23] V.J. Watson, B.E. Logan, Analysis of polarization methods for elimination of power overshoot in microbial fuel cells, Electrochem. Commun. 13 (2011) 54-56. doi:10.1016/j.elecom.2010.11.011.

[24] J. Winfield, I. Ieropoulos, J. Greenman, J. Dennis, The overshoot phenomenon as a function of internal resistance in microbial fuel cells, 81 (2011) 22-27. doi:10.1016/j.bioelechem.2011.01.001.

[25] X. Zhu, J.C. Tokash, Y. Hong, B.E. Logan, Controlling the occurrence of power overshoot by adapting microbial fuel cells to high anode potentials, Bioelectrochemistry. 90 (2013) 30-35. doi:10.1016/j.bioelechem.2012.10.004.

[26] Y. Hong, D.F. Call, C.M. Werner, B.E. Logan, Adaptation to high current using low external resistances eliminates power overshoot in microbial fuel cells, Biosens. 
Bioelectron. 28 (2011) 71-76. doi:10.1016/j.bios.2011.06.045.

[27] Y. Tan, W.H. Moase, C. Manzie, D. Nešić, I.M.Y. Mareels, Extremum seeking from 1922 to 2010, in: Proc. 29th Chinese Control Conf., 2010: pp. 14-26.

[28] M. Krstić, Performance improvement and limitations in extremum seeking control, Syst. Control Lett. 39 (2000) 313-326. doi:10.1016/S0167-6911(99)00111-5.

[29] K.B. Ariyur, M. Krstic, Real Time Optimization by Extremum Seeking Control, John Wiley \&amp; Sons, Inc., New York, NY, USA, 2003. 